\title{
Participación social en salud en la atención primaria en Bogotá: voces de mujeres*
}

\section{Social participation in primary health care in Bogota: Women's voices}

\section{A participação social em saúde na atenção primária em Bogotá: Vozes das Mulheres}

Fecha de recepción: 27-09-13 Fecha de aceptación: 06-03-14

doi:10.11144/Javeriana.RGYPS13-26.pssa

Janeth Carrillo Franco**

Lucero López***

Artículo de investigación derivado de la tesis doctoral titulada Mujeres participando y construyendo: La participación social en la atención primaria, Doctorado en Salud Pública, Universidad Nacional de Colombia, 2010-2012.

** Médica, doctora en Salud Pública. Subdirectora de Educación Médica e Investigación, Hospital Universitario de la Samaritana, Bogotá ( Colombia). Correspondencia: Cra. 51 No. 75-14, Bogotá (Colombia). Correo electrónico: 


\section{Resumen}

Objetivo: describir las dinámicas culturales en la participación social en salud de un grupo de mujeres de la localidad de Chapinero, Bogotá (Colombia). Materiales y métodos: investigación cualitativa con historia oral, con seis mujeres líderes comunitarias en Bogotá. El análisis fue inductivo e interpretativo. Resultados: se presenta el tema cultural: mujeres participando y construyendo un paraíso posible, una categoría y dos subcategorías. En el tema cultural se revela la relación y tensión de la organización de mujeres migrantes por alcanzar el sueño de legitimar la propiedad, tener oportunidades económicas, hacer cambios en sus vidas y las respuestas institucionales. Los significados culturales van cambiando, mientras las instituciones mantienen un lenguaje técnico y jerárquico. Conclusión: la comunidad relaciona la autoorganización para la resolución de sus necesidades con la participación social en salud. Por tanto, las dinámicas culturales deben ser consideradas para la interlocución con los procesos comunitarios.

Palabras clave: participación social; atención primaria en salud; cultura; dinámicas culturales; historia oral

Palabras clave descriptor: participación social; atención primaria de salud; cultura; historia oral

\section{Abstract}

Objective: Describe the cultural dynamics in social participation in health of a group of women from Chapinero, Bogota - Colombia. Materials and methods: Qualitative research with oral history was developed with six women, community leaders in Bogotá. Were analyzed inductively and interpretative. Results: We present the cultural theme: Women participating and building a possible paradise, a category two subcategories. In the cultural theme of the relationship and tension organizing migrant women to achieve the dream of legitimizing the property, have economic opportunities, make changes in their lives and the institutional responses is revealed. Cultural meanings are changing, while the institutions maintain a technical and hierarchical language. Conclusion: The community self-organization relates to solve your needs with the social participation in health. Therefore, Cultural dynamics should be considered for dialogue with community processes.

Keywords: Social participation; primary health care; culture; oral history

Keywords plus: social participation, primary health care, culture, oral history

\section{Resumo}

Objetivo: descrever a dinâmica cultural da participação social em saúde de um grupo de mulheres da cidade de Bogotá (Colômbia). Materiais e métodos: pesquisa qualitativa com a história oral, com seis mulheres líderes comunitárias em Bogotá. A análise foi indutivo e interpretativo. Resultados: A questão cultural é apresentada: as mulheres que participam e constroem um possível paraíso, uma categoria de duas subcategorias. No tema cultural revela da relação e tensão a organização as mulheres migrantes para realizar o sonho de legitimar a propriedade, ter oportunidades econômicas, fazer mudanças em suas vidas e as respostas institucionais é revelado. Significados culturais estão mudando, enquanto as instituições mantêm uma linguagem técnica e hierárquica. Conclusão: comunidade auto- organização se relaciona para resolver as suas necessidades com a participação social em saúde. Portanto, as dinâmicas culturais devem ser considerados em diálogo com processos comunitários

Palavras chave: participação social; atenção primária à saúde; cultura; história oral Palavras chave descritores: participação social, atenção primária à saúde, cultura, história oral 


\section{Introducción}

Como faceta de la participación cívica general, la participación social en salud (en adelante PSS) se considera una condición para ejercer la libertad, la democracia, el control social sobre la acción pública, y por lo tanto, para la equidad. Es una condición esencial para garantizar la efectividad y la satisfacción en los servicios y constituye un fin deseable en sí mismo (1). Sin embargo, como lo menciona Menéndez, no es un tópico propuesto desde y para la salud ya que "la participación social [...] era parte de las formas de actuación colectiva de la sociedad, especialmente de los grupos políticos y sindicales" (2). La mayoría de ejercicios de PSs se genera en lugares e intereses externos a las comunidades, quienes son el objeto de esta y no sus sujetos, aunque proliferen cientos de organizaciones populares fraccionadas, aparentemente participantes.

En la revisión de 127 artículos realizada por Tavares et ál. (3) se identifican tres abordajes en PSs: el ideal, definido como la capacidad de los individuos de influenciar o intervenir en las decisiones políticas de la sociedad en la cual viven, como parte de un derecho positivo que fomenta el capital social. El segundo, muestra un carácter político como medio de democratización del Estado y la definen como una de las herramientas de los gobiernos para evaluar sus capacidades gerenciales. El tercero, es instrumental, asociado con el concepto de medicina comunitaria de Estados Unidos, donde la participación de individuos, familias y comunidades se ha convertido en una herramienta para que compartan con el Estado la responsabilidad por la atención en salud.

La PSS instrumental se identifica con el conjunto de actividades que justifican los programas institucionales, definido lo institucional como ideología, normas y estructura (3). Es característico de las agencias internacionales, cuya prioridad se resume en que los beneficiarios puedan "escoger" entre opciones de oferta en un contexto de mercado. De esta manera, "la participación social como concepto y como práctica fue (es y sigue siendo) utilizada por tendencias teórico/ideológicas que consideraron que el capitalismo favorece el desarrollo de una sociedad individualista, competitiva, consumista, pasivo/receptiva, apática, donde los objetivos privados se imponen a las necesidades públicas" (2).

La Atención Primaria en Salud (en adelante APS) como estrategia y como modelo de atención plantea la participación social como el derecho y la capacidad de la población a participar efectiva y responsablemente en las decisiones del cuidado de la salud y su implementación (1). En la corriente de renovación de la APs se tomó en cuenta que la mayoría de las experiencias con enfoque integral han mostrado resultados positivos sobre el estado de salud de las poblaciones en países donde es considerada centro de la estructura del sistema de seguridad social y puerta de entrada a este: España (4,5), Canadá (6,7), Cuba (8), Costa Rica (9), Nueva Zelanda $(6,10)$.

En América Latina, varios países implementaron la APs junto con reformas en sus sistemas de salud. Es así como Costa Rica, Cuba, Chile y Brasil han obtenido resultados importantes en el estado de salud (1). Sin embargo, en el marco de sistemas de salud no universales en países con regímenes políticos económicos capitalistas, sustentados en la desigualdad social, como lo menciona Franco (11), la APS se ha concebido como aquellos paquetes de servicios para pobres, con un carácter selectivo. La existencia de múltiples experiencias aisladas es un indicador de la falta de coherencia del modelo de atención propuesto desde la política con las necesidades de salud de la población, resaltando las consecuencias de la fragmentación y segmentación de los sistemas de salud donde se insertan (12). 
En Colombia, varios gobiernos locales, en coherencia con el movimiento de renovación de la APs propusieron modelos como los implementados en Santander (13), Valle (14), Antioquia (15) y Bucaramanga (16), entre otros, cuyo denominador común es la atención integral y la participación social como elementos articuladores para el logro de la equidad en salud. Por su parte, Bogotá implementó la Atención Primaria en el año 2003, trazando como meta en su plan de desarrollo "la universalización progresiva del derecho a la atención integral con enfoque familiar y comunitario" (17). Con ello se buscaba superar las contradicciones y los resultados generados a partir de la reforma en 1993 que oscilan entre la satisfacción por la cobertura y el financiamiento (18) y la preocupación por la inequidad y la concepción utilitarista de la salud, donde prevalece lo económico sobre lo humano (19).

Bogotá complementó su modelo aunando la estrategia promocional de calidad de vida y salud (en adelante EPCVs), cuyo imperativo ético era responder a las necesidades sociales, integrado al compromiso de la gestión distrital con la garantía de los derechos humanos y sociales y con el desarrollo de la ciudad como un espacio moderno y humanizado (20).

La conjunción de las orientaciones de la APS y de la EPCVs se dio en el marco de la primera administración de izquierda en Bogotá en el año 2004 (21), apoyado por varios representantes de la corriente de la medicina social latinoamericana. La propuesta conceptual de la estrategia se basó en el reto por desarrollar una nueva salud pública que se liberara del marco de la determinación biologicista y avanzara hacia una perspectiva más colectiva de la salud (22-24). Se pretendía que este ejercicio de APS se legitimara socialmente, se sustentara y tuviera permanencia en el tiempo a través de la participación de la comunidad, ejercicio propuesto con una clara perspectiva de desarrollo humano y comunitario (25), afirmándose como espacio y oportunidad de empoderamiento para ejercer el derecho de participación (26).

Se trataba de superar la racionalidad política neoliberal que organizó los servicios de salud en torno a un modelo de mercado y definió, desde el sistema de salud, dos tipos de participación en el sistema: individual (para velar por la calidad de los servicios) y colectiva (para ejercer la función de control social). La estrategia se implementó a través del programa Salud a su $\operatorname{Hogar}^{1}$ (SASH), dirigida a población vulnerable de la ciudad (estratos ${ }^{2}$ 1 y 2), conformando equipos básicos con un médico, una enfermera y dos promotores de salud, para la atención de 1200 familias referenciadas geográficamente en espacios denominados microterritorios, compuestos por barrios que tienen una cercanía geográfica. Finalizando el año 2011, existían más de 425 territorios distribuidos en la mayoría de las 20 localidades de la ciudad. A partir de ese año, el programa sufre un ajuste operativo, definiendo desde entonces un territorio como aquel conformado por cinco de los antiguos microterritorios y 6000 familias a cargo de un equipo básico (25).

Las dificultades encontradas para el desarrollo de la PSS propuesta por la APS en el marco fragmentado del sistema de seguridad social en salud en Bogotá son similares a los hallazgos para la participación en general en el país. Las escasas investigaciones en el área concluyen que hay pobre acceso a la

1 A partir de 2008 -bajo la administración distrital del alcalde Samuel Moreno- el programa cambió su nombre a Salud a su Casa (SASC).

2 Nivel de clasificación de la población de acuerdo al grado de riqueza y calidad de vida, determinada de manera indirecta mediante las condiciones físicas de las viviendas y su localización. Estrato 1: bajobajo, estrato 2: bajo (Ley 142 de 1994, Art. 102). 
información, bajo liderazgo comunitario en el control social, resistencia de los funcionarios públicos a que su gestión sea objeto de control, falta de cultura participativa y pobres resultados en la interacción de la comunidad con las instituciones (27-29). En el caso particular de Bogotá y para el ejercicio de Pss en el marco de Salud a su Casa, Restrepo (30) realizó entrevistas a profundidad y grupos focales con equipos de APS de la localidad de Suba, y concluyó que "Bogotá sigue utilizando la participación fundamentalmente para promover y vender servicios; para lograr objetivos, metas y rentabilidades; y como medio para legitimar discursos y programas, para así obtener recursos".

De esta manera, se evidencia cómo la participación planteada para la ciudad y para los territorios de APS se redujo a un activismo instrumentalizado. Al parecer, no se ha logrado superar la aplicación de las mismas rutinas y actividades operativas de pss que desde la mirada de la comunidad pueden estar desprovistas de sentido para quienes va dirigida.

A nuestro juicio, la Pss continúa partiendo del paradigma y desde las jerarquías de las instituciones, sin tener en cuenta las dinámicas culturales de la comunidad. Tanto esta como los miembros de las instituciones viven y provienen de grupos permeados por diferentes dinámicas culturales como el lenguaje, la educación, la socialización primaria en la familia, el etnocentrismo, las relaciones de poder o el género (31).

Bajo esta consideración, se toman en cuenta dos dinámicas culturales concurrentes: la del sujeto formado en una determinada cultura que puede acercarse a la Pss dotándola de sentido desde la socialización que haya tenido. La de la misma participación como dinámica cultural, imbricada de significados asignados en el devenir de la formación del territorio o de la comunidad, de la respuesta institucional, de la memoria de estos contactos y de su efectividad en la resolución de las necesidades. La participación está ligada al proceso de "construcción de comunidad e implica relaciones del individuo con el ecosistema en que éste está inmerso, generando experiencia, conocimiento y praxis que posteriormente se incorpora a la historia de la comunidad y forma parte de la cultura" (32).

Entonces, la relación individuo-ecosistema influye en la participación pero hay factores de los profesionales y de los formuladores de política que pueden considerarse como barreras para esta. Para el usuario se identifican conocimientos, actitudes, grado de organización y cohesión. Para el personal de salud, la interacción entre actores en desigualdad de poder, mientras que para los formuladores de política, factores relacionados con las instituciones, como el temor del personal -tanto público como privado-a responder a las críticas de la comunidad; la limitada capacitación e información que las instituciones proporcionan a los usuarios y la ausencia de voluntad para abrir espacios participativos, especialmente en la toma de decisiones (28).

Si la APs parte del abordaje de la participación como derecho, es imprescindible un enfoque cultural que permita su implementación flexible y heterogénea, desde la formación de equipos capaces de "tomar en consideración las percepciones, creencias y necesidades de los usuarios con el objetivo de optimizar los resultados, así como de evitar los efectos negativos que emergen de los flujos de información incorrectos, del abismo que se da entre las metas institucionales y las de los usuarios, así como del suministro de servicios que no conceden un rol activo a los usuarios" (33).

Ello implica la reivindicación de lo comunitario, de la recuperación de la voz del otro, la comunidad "desde" no "para" ellos. Las 
dinámicas culturales son menos reconocidas a nivel de la literatura y de la investigación en salud pública y se desestiman las repercusiones en el acercamiento institucional a la comunidad. Todo esto sumado a la ausencia de incentivos para mantener la participación y la precaria formación en cultura democrática que tienen las comunidades de base, la población en general y los profesionales en salud, produce la resistencia al cambio de los actores involucrados. Bajo estas consideraciones, el objetivo del artículo es describir las dinámicas culturales en la participación social en salud de un grupo de mujeres de la localidad de Chapinero, Bogotá (Colombia).

\section{Materiales y métodos}

La investigación cualitativa se desarrolló a través de la historia oral, siguiendo la metodología del oralista brasileño Jose Carlos Bom Meihy. Así, fue una herramienta para captar la historia en tiempo presente -historia viva- referida a la experiencia social de personas y grupos, para responder a una necesidad de utilidad práctica, social e inmediata (34). De esta manera, se dio voz a las colaboradoras, fue posible visibilizar las dinámicas culturales de acuerdo con quienes las viven, y a sus experiencias cotidianas.

La selección del territorio de San Luis fue emblemática, en términos de la participación social, debido a: 1) su aislamiento relativo en los cerros orientales. El territorio surgió del loteo de una hacienda de mayor extensión en la década de los setenta. Por su origen rural, la prestación de servicios públicos era limitada y la búsqueda del agua llevó a la construcción de su propio acueducto, que actualmente es dirigido por la comunidad; 2) los habitantes comparten los procesos migratorios campo-ciudad verificados durante la década de los ochenta en en busca de nuevas oportunidades; 3) En la década de los ochenta, la Fundación Santa Fe realizó un trabajo de salud comunitaria relacionado con sus objetivos misionales que involucró a la mayoría de la población. Este constituyó un hito en la atención en salud y en la organización comunitaria del territorio, ya que involucró de manera permanente a la comunidad.

4) A pesar de la venta legal de los lotes, por normatividad relacionada con la protección ambiental de los cerros orientales, el territorio quedó ubicado en la zona de reserva forestal, lo que hace que se constituya en un territorio ilegal para las instituciones. Este tema en particular convoca la participación de manera permanente de los pobladores del territorio, ya que las autoridades siguen sin definir el uso de las viviendas construidas en la reserva antes de su declaratoria como tal.

El método requirió la definición de la colonia y la red. La primera, entendida como un aspecto preponderante de un conjunto de personas y la red como el segmento de la colonia que se desea estudiar (34). La colonia fue representada por hombres y mujeres mayores de edad, pobladores del territorio de APS San Luis en los cerros orientales de Bogotá, que vivieran en el territorio desde su poblamiento e identificados como líderes desde la institucionalidad (el hospital de Chapinero). Inicialmente, fueron contactados ocho líderes (cuatro mujeres, cuatro hombres). De los hombres convocados, uno aceptó participar. Sin embargo, no tuvo disponibilidad de tiempo para conceder las entrevistas.

Dada la proximidad de una de las investigadoras con las mujeres líderes en la comunidad, fueron contactadas cuatro mujeres líderes, denominadas como la primera generación por ser mujeres mayores de 60 años y primeras pobladoras del territorio. Después del análisis de la primera generación de lí- 
deres, la red fue nutrida con dos líderes más jóvenes quienes nacieron en el territorio y fueron mencionadas por las líderes más antiguas. A ellas se les llamó segunda generación.

Las entrevistas fueron realizadas entre septiembre de 2010 y diciembre de 2011. Los espacios fueron concertados con las mujeres, generalmente el domicilio. Hubo varios encuentros con duración de 40 a 90 minutos. Todas las entrevistas fueron transcritas y, de acuerdo con Meihy, se realizó la textualización y transcreación (34). El primero, consistió en el paso de lo oral a un documento escrito mediante un proceso donde se pasó la narrativa a primera persona del singular, se incorporaron las preguntas al texto, cuidando el universo vocabular del entrevistado y eliminando repeticiones propias de lo oral. La historia fue organizada en una secuencia lógica y da relevancia a contenidos propios del eje temático de la investigación.

Posteriormente, se realizó la transcreación, que consiste en convertir el texto en un relato literario atractivo, donde el investigador es parte de su creación. Se identificó el "tono vital" -frase que muestra la esencia individual de la colaboradora-, lo que involucra un primer ciclo de análisis. Ese documento se fue enriqueciendo con las nuevas entrevistas. Se tuvo entre seis y ocho versiones de la historia. La versión final se obtuvo después de presentar la historia transcreada a cada colaboradora y luego de que esta fuera realimentada por ellas confirmando, ampliando o complementando información, y así se obtuvo la historia definitiva (de allí se toman los apartes presentados en el texto).

El proceso de análisis se desarrolló en dos grandes momentos: en la construcción de la historia oral en sí misma como, fue anotado anteriormente, y posteriormente se realizó el análisis por edición (35), por medio del cual se desarrolló un esquema de categorización con las historias transcreadas que fueron analizadas de forma individual y luego contrastadas en conjunto. Inicialmente entre las líderes de la primera generación y posteriormente con las líderes de la segunda. El proceso se auxilió con el uso se de esquemas. En los diferentes procesos hubo discusión y consenso entre los investigadores sobre las categorías emergentes. Emergió un tema cultural, dos categorías y cinco subcategorías. Para efectos del presente artículo se presenta el tema cultural, una de las categorías con dos subcategorías.

Los aspectos éticos de la investigación están acordes con lo dispuesto en la Resolución 8430 de 1993. Contó con aprobación del Comité de Ética de la Facultad de Medicina de la Universidad Nacional de Colombia, mediante acta número 1 del 29 de enero del 2009. Las colaboradoras firmaron el consentimiento informado. Los nombres fueron escogidos por las colaboradoras, algunas decidieron dejar su nombre de pila.

\section{Resultados}

\section{Características de las mujeres}

La primera generación: cuatro mujeres entre los 62 y los 70 años, con primaria incompleta, provenientes de diversas zonas rurales del país y que viven en el territorio hace más de treinta años. La segunda generación: dos mujeres, de 26 y 37 años, con formación universitaria y que viven en el territorio desde su primera infancia.

Tema cultural: Mujeres participando y construyendo un paraíso posible

El tema cultural indica la relación que tiene para las mujeres la organización comunitaria para obtener un lugar apropiado para vivir, donde se van a tejer sueños de prosperidad 
y se hará realidad su paraíso. Para el logro de este paraíso, las mujeres "resuelven la cotidianidad", su día a día de necesidades, buscando el acceso a servicios públicos y tomando de las instituciones aquello que les es útil a esos propósitos.

Se puede conocer el carácter de las líderes y su búsqueda del paraíso posible mediante el tono vital (tabla 1) de cada una -frase resumen de una historia de vida y de la mirada particular sobre la Pss-. Los tonos vitales marcan a las mujeres de la primera generación en una dinámica cultural que está centrada en conquistar el territorio, obtener lo necesario para sobrevivir y lograr el sueño de un futuro mejor en la ciudad. Para las mujeres de la segunda generación la participación es una herencia con objetivos diferentes. Aunque aparentemente son menos aguerridas que las de la primera generación, el contexto de necesidades no es igual, pero siguen distinguiéndose por su participación a favor de beneficios para la comunidad.

\section{Categoría: Resolviendo la cotidianidad en busca del paraíso posible}

El paraíso posible tiene origen para cuatro de las mujeres en la migración del campo a la ciudad, desde diferentes zonas del país
(Boyacá, Antioquia, Huila), por el conflicto armado o tras nuevas oportunidades, laborales, familiares, de atención en salud y áreas rurales atraídas por Bogotá como una zona de impulso económico y con posibilidades para hacer un cambio en las condiciones de vida.

La dinámica cultural básica se centra en la propiedad de la tierra. Siendo un territorio ubicado en la reserva forestal de los cerros, los asentamientos son declarados ilegales por las instituciones de la ciudad. Sin embargo, cuando las mujeres y sus familias lo escogen, sobre toda dificultad se antepone la necesidad de legitimar la propiedad de la tierra. Siendo migrantes de origen campesino, el territorio reúne a otros como ellos con un poder adquisitivo reducido. El territorio es aquello que es "valioso para otros pero que está ocupado arbitrariamente por las personas equivocadas, que no pueden invertir de manera adecuada para hacerlo más rentable" (36).

En este lugar había pocas casas, pero había vegetación y mucho monte nativo. Era muy bonito pero también era difícil: cuando llegué no había transporte. iImagínese! (Adelina)

TABLA 1. Tono Vital de las historias de VIDA de líderes comunitarias, SAN Luis, territorio de aPs, LOCALIDAD DE CHAPINERO

\begin{tabular}{|c|c|}
\hline Colaboradora & Tono vital \\
\hline \multicolumn{2}{|r|}{ Primera generación } \\
\hline Adelina & Fuimos las mujeres las que sacamos todo adelante \\
\hline Lucía & A mí nunca me ha gustado quedarme dormida, desde niña participaba en todo \\
\hline Mery & Las mujeres éramos muy unidas \\
\hline María Luisa & El día que usted no aprende algo, ese día se perdió \\
\hline \multicolumn{2}{|r|}{ Segunda generación } \\
\hline María Lucía & Participar con la comunidad es una opción personal \\
\hline Jennifer & Les digo a las mujeres que tienen que transformarse \\
\hline
\end{tabular}


Estamos en un paraíso, no quiero decir que aquí es perfecto, súper sano pero se vive casi que en un $90 \%$ de tranquilidad comparado con otras zonas y con otro sector de la ciudad. (Lucía)

Los miembros de la comunidad descubren que aun cuando estos paraísos pueden tener matices diferentes en sus significados, es común la expectativa de legitimar la pertenencia del territorio para todos ellos. Ello implica que dinámicas como transmitir la cultura de participar promueva la propia organización comunitaria:

Me gusta vivir aquí, porque es muy tranquilo, a pesar de todo. El aire que nosotros respiramos no lo respira nadie en la ciudad, eso le puedo asegurar y mi hogar no lo cambio por nada. (María Luisa)

Cuando nos pasamos no había luz, agua, teléfono, ni transporte. Aún con todas estas dificultades, inosotros compramos! (María Luisa)

Sin embargo, el paraíso debe construirse. Las mujeres parten de necesidades apremiantes que deben resolver en lo cotidiano, encontrando que de manera colectiva se logran mejores resultados. La voz de las mujeres que ratifica esta definición se genera a partir de la construcción de respuestas a sus necesidades de servicios públicos, que las afectan de manera particular, ya que son quienes afrontan la cotidianidad desde las carencias. Las mujeres enfrentan las necesidades del día a día con una respuesta conjunta que terminará siendo el motor de la organización comunitaria.

Cuando nos pasamos no había luz, agua, teléfono, ni transporte. Aún con todas estas dificultades, inosotros compramos! Traíamos el agua de un nacedero, era muy pura. Después, las juntas directivas se organizaron para lo del acueducto. Nos transportábamos en la flota Valle de Tenza o a veces bajábamos y subíamos a pie porque la flota cumplía su ruta con un horario. (María Luisa)

Como necesitábamos otros servicios, empezamos a reunirnos en comités, como para conseguir el agua. Cuando nos organizamos tuvimos bastante apoyo: nos propusieron que nos organizáramos para hacer un tanque de almacenamiento arriba y que nos traían el agua hasta las casas, que hiciéramos un acueducto comunitario, porque el de Bogotá no lo podíamos tener porque el barrio no era legal. (Adelina)

La organización por el agua identifica de manera contundente el embrión del proceso de desarrollo comunitario, considerándose una de las necesidades más sentidas, cuya ausencia surcaba de manera transversal la cotidianidad de las mujeres:

Éramos las mujeres las que estábamos ahí. iClaro! como nos tocaba la lucha para el agua de la cocina, del baño, de lavar, nosotras nos movíamos. (Adelina)

Había un pozo descubierto aquí cerca, a donde teníamos que ir a la media noche, pero era agua muy limpia porque casi no había familias. Como no había Junta de acción Comunal, se crearon unos comités y se trabajó con un proyecto, que proponía traer agua del Verjón. (Lucía)

Durante el desarrollo de la investigación se evidenció que desde la perspectiva de la historia oral de las mujeres participantes, la PSs puede asimilarse a la organización comunitaria, cuyo motor es la búsqueda del bienestar propio y del entorno. Fue sorprendente porque pese a las diversas formas de indagación y el conocimiento que se iba construyendo del grupo de colaboradoras, el término "participación social en salud" no se encuentra en su discurso, ni hay uno equivalente. Para ellas, la 
organización se da a partir de las necesidades cotidianas en un territorio cambiante pero valorado como un paraíso posible.

\section{Subcategoría 1: las jerarquías en salud}

Del otro lado, las instituciones que lideran la pss en el territorio parecieran asumir que la relación con la comunidad se genera en espacios sin una construcción histórica, permeados de significados, interpretaciones y reinterpretaciones. Se constata una brecha mediada por la cultura entre los operadores (médicos, enfermeras, personal de salud), de un lado, y los sujetos a quienes va dirigida (población en general), del otro.

Sin embargo, la organización comunitaria lleva a las mujeres a relacionarse con las instituciones del territorio. Comprendieron que a través de ellas y de sus objetivos pueden acercarse de manera más contundente a las respuestas para sus necesidades:

La necesidad de antes fue la que nos llevó a meternos de lleno con las instituciones. (Mery)

Sin embargo, la relación comunidad-institución no está exenta de tensiones, ya que la institución tiene sus propias dinámicas culturales que la diferencian como grupo: conocimientos, lenguaje, educación, etnocentrismo, relaciones de poder (31), que son especialmente marcadas en el ámbito de profesionales de la salud:

La gente participa en las reuniones pero le gusta que sea rápido, está cansada de tanta reunión, ya no cree porque es mucho tilín tilín y inada de paletas! (Mery)

Hoy la gente no participa como hacíamos de jóvenes. Es muy apática, los presidentes de las juntas son los mismos, o a las reuniones siempre van los mismos. Creo que es falta de conciencia de la gente, les gusta mucho criticar y decir que los otros no hacen, que se robaron la plata, pero nadie se mete en el cuento. (María Lucía)

La falta de respuesta institucional a sus necesidades se constituye en una barrera para la PSS. Para las mujeres se hace evidente la verticalidad institucional y el desconocimiento de las dinámicas culturales, ya que se perciben que las instituciones se encuentran alejadas de su propia lectura de necesidades.

Hoy en día, los proyectos son muy cortos y la idea dura lo que dure al alcalde o la organización o el concejal de turno. No son unos proyectos de continuidad, es decir, esto se hace, se continúa y hay que seguirlo. Lo de Salud a su Casa está bien para que la gente se dé cuenta, tanto los de allá, como los que viven acá, porque es muy difícil diagnosticar una comunidad desde un escritorio. (Mery)

\section{Subcategoría 2: la participación como trans- misión cultural}

La cotidianidad cambia y otras generaciones en el territorio hacen frente a nuevas necesidades. Se han resuelto la mayoría de las necesidades iniciales: agua, servicios públicos, educación. Como lo hizo en el pasado, la comunidad organizada se inserta en esta trama de sentidos comunes, transmite sus saberes y experiencias exitosas como en el caso de la Pss:

Sí, precisamente para eso está uno educando a sus hijos, para que lo remplacen a uno (Adelina).

Generalmente veíamos a los hijos de los padres que trabajábamos en lo comunitario, y los muchachos estaban detrás de los papás y detrás de las mamás... (María Luisa) 
Cuando uno es un líder o cuando uno participa, los hijos salen: como en este momento sobre todo mis hijas; yo los llevaba a las reuniones, iimagínese?! El bebecito de 3, 4 años, le tocaba ir conmigo: -siéntese mijito que voy a dar una charla o que voy a dar un discurso [...] iQué risa!... (Mery)

Existe un aprendizaje vicario donde se socializa y se culturiza dentro de unos significados, uno de los cuales, la organización comunitaria, es parte del tejido que les permite obtener respuesta a sus necesidades.

Sin embargo, la baja participación hoy evidencia que la transmisión cultural se ve afectada por los ruidos de fondo de una sociedad cambiante, como los medios de comunicación, que debe regenerar sus pactos para la participación social en salud, así esta se considere una forma más de reproducción de la estructura social. En este orden de ideas, la dinámica cultural para la nueva generación debe contextualizarse en tiempos de respuesta más oportunos, tal cual lo perciben los jóvenes desde su perspectiva de inmediatez.

\section{Discusión}

Varias dinámicas culturales se relacionan con la PSs. Conocimientos previos, necesidades del territorio, actitudes de las instituciones hacia la comunidad, grado de organización y cohesión de la población, son punto de partida para la organización comunitaria, a través de la cual las colaboradoras intentarán conseguir su paraíso posible (28).

La misma participación actúa como dinámica cultural, imbricada de significados asignados en el devenir de la formación del territorio o de la comunidad, de la respuesta institucional, de la memoria de estos contactos y de su efectividad en la resolución de las necesidades. La resolución cotidiana de las necesidades, aún de las básicas como en el caso del agua para el territorio, genera un ejercicio cercano a la participación social. Sin embargo, desde la perspectiva jerárquica de lo institucional, la mayoría de las propuestas de participación en APs son limitadas para lo comunitario, ya que dichas instituciones no dan respuesta a las necesidades inmediatas o mediatas y pierden de manera automática su credibilidad, se refuerza esa desconfianza junto con el sentimiento de desamparo. Persiste la postura de ignorar que "la población escucha lo que quiere escuchar; escucha mejor aquello en lo cual tiene experiencia y no le interesa la manera en que nosotros (las instituciones) pensamos debe hacerse" (37).

La búsqueda de alternativas para la resolución de la cotidianidad conlleva relacionarse con las instituciones presentes en el territorio, desde la negociación de la necesidad comunitaria y la necesidad institucional. Esta última enmarcada en jerarquías, una de las cuales está representada por los profesionales, quienes poseen un lenguaje técnico que los separa de sus pacientes -su propio cuerpo de conocimientos-, enfatizan en la enfermedad y se basan de manera frecuente en la tecnología. Son ellos quienes lideran los procesos de participación social en salud, desconociendo que son parte del lugar donde ejercen, que a su vez es parte de un sistema mayor de creencias, comportamientos y actitudes (31).

El tema de las jerarquías institucionales constituye una manifestación de lo que Fassin denomina la "ideología dominante", que reduce "la salud simplemente a la medicina y del otro a la manera como los médicos han logrado obtener el monopolio de la actividad terapéutica legítima, donde la verdadera actividad de participar, opinar y decidir en salud es considerada como una amenaza a 
un cierto grado de pérdida de poder, siendo denunciada en nombre de la complejidad del saber médico" (38).

Así, de un lado las dinámicas culturales que brindan significados al territorio no son tenidas en cuenta por la institucionalidad. De otro lado, la misma comunidad no logra identificarse a sí misma como lo hizo en el pasado. Nuevos significados culturales y sociales son atribuidos al territorio, ya que se "han acabado la mayoría de los puntos de referencia constantes y sólidamente establecidos que sugerían un entorno social más duradero, más seguro y más digno de confianza que el tiempo que duraba una individualidad" (39). La comunidad organizada inserta en esta una trama de sentidos comunes, intenta transmitir sus saberes y experiencias exitosas a las siguientes generaciones.

Como lo manifiesta Spector (40), “aprendemos de nuestro propio pasado cultural y étnico". Sin embargo, recuperar esta mirada de las dinámicas culturales de las mujeres del territorio es muy relevante en el marco de la Atención Primaria, para la cual la "comunidad" se transformó en el corazón de la política sanitaria mundial. "Gracias a la dimensión de consenso que en sí misma encierra, poniendo de relieve lo que acerca a los individuos, lo que tienen 'en común', más que lo que les opone, la representación comunitaria del mundo social se presta particularmente bien a la utilización política que de ella hacen las instituciones internacionales" (37). Las dinámicas culturales, por tanto, deben ser tenidas en cuenta para la interlocución con los procesos comunitarios que quieren fortalecerse.

\section{Conclusiones}

La PSS está permeada por dinámicas culturales complejas, cambiantes entre generaciones y en el territorio. Desde lo institucional queda reducido a una serie de actividades operativas, dado el abordaje instrumental del sector salud, para el cual su principal finalidad es la justificación de su modelo de atención. Uno de los aspectos relevantes para la política pública es el de recuperar la voz del interlocutor institucional, en este caso las mujeres, cuya voz se deja viva y se recupera como fuente documental, mujeres quienes de forma aguerrida y desafiante dejan ver las dinámicas culturales de su comunidad, las diversas tensiones de los actores en el territorio y el movimiento histórico en este.

En el ámbito operativo, evidencia las tensiones propias del modelo biomédico y de las jerarquías en salud, cuyos representantes están imbuidos de modelos organizativos y culturales rígidos que desde la formación de sus profesionales les asignan roles específicos y los terminan distanciando de la comunidad. Para el periodo analizado y en el territorio descrito, el estudio muestra la dificultad de implementación de APS para Bogotá, porque persiste la mirada institucionalizada y vertical desde el nivel central (Secretaría de Salud) y la PSs se plantea como una acción sistemática y similar para la ciudad en general, sin tener en cuenta las particularidades territoriales, comunitarias y culturales y sin dotar a los equipos de APs de la capacidad de decisión en lo local.

Los resultados de esta investigación hacen evidente la necesidad de reconocer que hay dinámicas culturales particulares en el interior de los territorios sociales que permiten la elaboración de proyectos particulares que fomenten la participación, así como la reflexión sobre las jerarquías del modelo biomédico a través del cual se pretende implementar modelos de atención como la APs renovada. Una de las limitaciones del proyecto de investigación fue considerar el punto de vista de las líderes. Por ello, serán necesarios estudios donde se pueda hacer contraste 
entre los equipos de APs y los formuladores de política. Será muy útil "incentivar trabajos investigativos con ésta perspectiva donde participen diversos profesionales y grupos en diferentes áreas rurales, urbanas, niveles socioeconómicos y culturales" (41).

La PSS requiere una apropiada preparación de los profesionales que la desarrollan, siendo uno de los primeros pasos superar afirmaciones y actitudes como aquellas que consideran que el trabajo comunitario requiere que se modifiquen solo aquellas de la comunidad cuando la principal resistencia al reconocimiento de las dinámicas culturales está en el personal de salud, ya que "a menudo aquellos cuyas actitudes y enfoque tradicional son más difíciles de modificar no son los aldeanos sino los profesionales" (38).

\section{Referencias bibliográficas}

1. Organización Panamericana de la Salud (ops). Renovación de la atención primaria de salud en las Américas. Un documento de posición de la Organización Panamericana de la Salud (OPS), Washington, D. C., 2005

2. Menéndez E. De sujetos, saberes y estructuras.. Buenos Aires: Lugar Editoria; 2009.

3. Tavares MLC, Henriques MPC, Rodrigues LA. Participação em saúde: uma sistematização de artigos ublicados em periódicos brasileiros. Ciência \& Saúde Coletiva. 2012; 17 (7): 1813-23.

4. Starfield B. Atención primaria y responsabilidades de salud pública en Europa y América del Norte. Revista Española de Salud Pública. 2004; 78 (1).

5. Larizgoitia I, Starfield B. Reform of primary health care: The case of Spain. Health Policy. 1997; (4): 121-37.

6. Starfield B, Shi L, Macinko J. Contribution of primary care to health systems and health. The Milbank Quarterly. 2005; 83 (3): 457- 502.

7. Blumenthal D, Mort E, Edwards J. The efficacy of primary care for vulnerable populations groups. Health Services Research. 1995; 30 (1).

8. Jiménez L. Metodología de la evaluación de la calidad de la atención en instituciones de atención primaria de salud. Revista Cubana de Salud Pública. 1996; 1.

9. Rosero Bixby L. Evaluación del impacto de la reforma del sector de la salud en Costa Rica mediante un estudio cuasiexperimental. Pan American Journal of Public Health. 2004; 15 (2): 94-103.
10. Hedfford M, Crampton P, Foley J. Reducing health disparities through primary care reform: The New Zealand experiment. Health Policy. 2005; 9-23.

11. Franco Giraldo A. Atención primaria en salud: ¿de regreso al pasado? Sexto Congreso Internacional de Salud Pública, 11 a 13 de junio de 2009. Medellín, Centro de Convenciones Plaza Mayor, Facultad Nacional de Salud Pública.

12. Bursztyn I, Kushnir R, Giovanella L, Stolkiner A, Sterman-Heimann L, Riveros M, Sollazzo A. Notas para el estudio de la Atención Primaria en contextos de sistemas de salud segmentados. Revista de Salud Pública. 2010; 12 (1): 77-88.

13. Acosta N. La implementación de la estrategia de APS en Santander, después de la reforma: logros, retos, propuestas de ajuste y líneas de acción. Universidad Industrial de Santander, julio de 2010 [internet] [acceso: septiembre de 2011]. Disponible en: http://www.consultorsalud.com/biblioteca/ documentos/2011/APS\%20Experiencia\%20de\%20 Santander.pdf

14. Arias Castillo L. Formación en APs para la reconstrucción del tejido social en salud. Sexto Congreso Internacional de Salud Pública, 11 a 13 de junio de 2009, Medellín, Centro de Convenciones Plaza Mayor, Facultad Nacional de Salud Pública [internet] [acceso: septiembre de 2012].

15. Vallejo A. Experiencia de implementación de una política pública en salud: Atención primaria con enfoque familiar y participación comunitaria [en línea] [acceso: noviembre de 2011]. Disponible en: http://guajiros.udea.edu.co/fnsp/congresosp6/memorias6/Viernes\%2012/Presentacion\%20Inv\%20 Comision\%205/Manana/P2.pdf

16. Instituto de salud de Bucaramanga. Isabu en su barrio [internet] [acceso: mayo de 2012]. Disponible en: http://www.bucaramanga.gov.co/documents/ PlanDeGobierno.pdf

17. Acosta Navarro O. Análisis de las políticas de educación, salud y vivienda del nuevo plan de desarrollo de Bogotá. Bogotá: Fundación para la Educación Superior y el Desarrollo; 2005.

18. Agudelo Calderón CA, Cardona Botero J, Ortega Bolaños J, Robledo Martínez R. Sistema de salud en Colombia: 20 años de logros y problemas. Ciencia \& Saude Coletiva. 2011; 16 (6): 2817-28.

19. Eslava-Schmalbach J, Guarnizo-Herreño CC, Hernández-Álvarez ME. Equidad en salud: posiciones e implicaciones. Revista de Salud Pública. 2008 Dic.

20. Secretaría Distrital de Salud. Salud a su hogar: un modelo de atención primaria en salud para garantizar el derecho a la salud en Bogotá. Bogotá: Secretaría Distrital de salud; 2004.

21. Luis Garzon: Un alcalde histórico. Revista Semana. 2003 Oct. 27.

22. De Negri AF. Adoção de uma estratégia promocional da qualidade de vida e saúde: transetorialidade das políticas públicas. En: Girotti Sperandio AMO. Proceso de construção da rede de municipios potencialmente saudáveis. Campinas-São Paulo: IPES, Unicamp, ops/oms; 2004.

23. De Negri AF. La construcción de alternativas políticas en pro del derecho integral a la salud. 
Bases conceptuales del ejercicio en la Secretaría Distrital de Salud de Bogotá. Investigaciones en seguridad social y salud. Bogotá: Secretaría Distrital de Salud; 2006.

24. Torres M. El reto por desarrollar una salud pública en contraposición a una salud privada. Revista Cubana de Salud Pública. 2007; 7: 33 (4).

25. Secretaría Distrital de Salud. Lineamientos Salud a su Casa, Prácticas favorables para la salud de las familias, 2011 [internet] [acceso: marzo de 2011]. Disponible en: http://saludpublicabogota. org/wiki/index

26. Torres M. Participación social: eje central de la renovación de la APs. La experiencia de Salud a su Hogar. Bogotá: Secretaría Distrital de Salud; 2005.

27. Arévalo D. Participación comunitaria y control social en el sistema de salud. Revista de Salud Pública. 2004. 6 (2): 107-39.

28. Delgado M, Navarrete L. Barreras y oportunidades para la participación social en Colombia: percepción de los actores principales. Revista Salud Pública. 2006; 8 (2).

29. Mosquera J, Gutiérrez A, Sierra M. La experiencia de participación ciudadana en el control social a la gestión en salud en Cali, Colombia. Colombia Médica. 2009; 40 (1).

30. Restrepo O, Vega R. Participación social y comunitaria en Atención Primaria de Salud (APS). Bogotá: para la muestra un botón. Revista Gerencia y Políticas de Salud. 2009; 8 (16): 153-64.

31. Helman C. Culture, health and illness. Oxford University Press; 2007.
32. Martínez KM. Comprensión del déficit de ciudadanía en Chile: la paradoja de su desarrollo. Revista Paraguaya de Sociología. 1999; 36: 105-22.

33. López L, Cataño N, López H, Velásquez V. Diversidad cultural de sanadores tradicionales afrocolombianos: preservación y conciliación de saberes. Aquichan. 2011; 11 (3): 287-304.

34. Bom JCM, Holanda F. Historia oral, como fazer, como pensar. Sao Paulo: Contexto; 2007.

35. Polit D, Hungler B. Investigación científica en Ciencias de la Salud, quinta edición. México: McGraw-Hill; 2000.

36. Peña Frade N. El territorio y las ciencias sociales: una relación cambiante y segmentada [en línea] [acceso: marzo de 2011]. Disponible en: http:// www.fuac.edu.co/recursos_web/descargas/grafia/ territor.pdf

37. Ugalde A. Las dimensiones ideológicas de la participación comunitaria. En: Participación social ¿para qué? Lugar, editorial. Buenos Aires, 2006.

38. Fassin D. Entre ideología y pragmatismo. Ambigüedades y contradicciones de la participación comunitaria en salud. En: Participación Social, ¿para qué? Buenos Aires: Lugar Editorial; 2006.

39. Bauman Z. Comunidad. En busca de seguridad en un mundo hostil. Madrid: Siglo XXI editores; 2006.

40. Spector R. Las culturas de la salud. Madrid: Prentice Hall; 2003.

41. López L, Cataño N, López H, Velásquez V. Efecto de un programa educativo para cuidadores de personas ancianas: una perspectiva cultural. Revista de Salud Pública. 2011. 13 (3). 


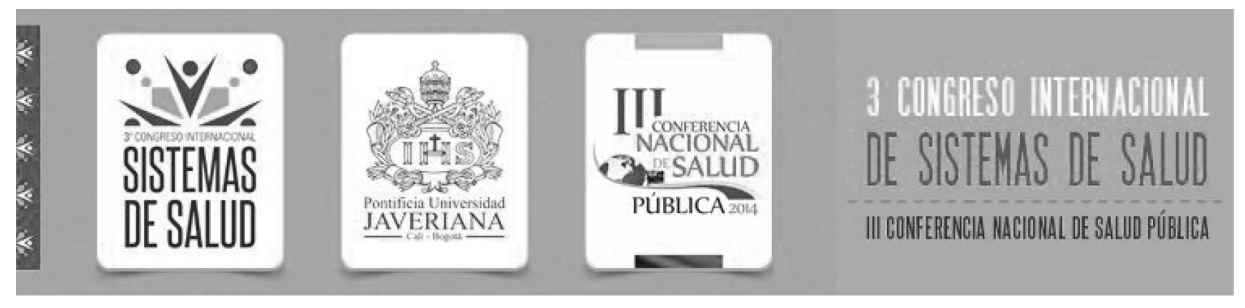

EQUIDAD, CALIDAD Y SERVICIOS DE SALUD

\section{CONVOVAN}

Programas de Posgrado en Administración de Salud y Seguridad Social Pontificia Universidad Javeriana Bogotá

Departamento de Salud Pública y Epidemiología

Especialización en Administración en Salud, Pontificia Universidad Javeriana Cali

Lugar y Fecha del Evento

Cali, Colombia, 1, 2 y 3 de octubre del 2014

\section{MAYOR INFORMACIÓN EN}

www.3congresosalud.com 